\title{
Further Stability Analysis of Time-Delay Systems with Nonlinear Perturbations
}

\author{
Jie Sun ${ }^{1}$ and Jing Zhang ${ }^{2,3}$ \\ ${ }^{1}$ School of Computer Science \& Software Engineering, Tianjin Polytechnic University, Tianjin 300387, China \\ ${ }^{2}$ Tianjin Vocational Institute, Tianjin 300410, China \\ ${ }^{3}$ School of Textiles, Tianjin Polytechnic University, Tianjin 300387, China
}

Correspondence should be addressed to Jing Zhang; chufei726@163.com

Received 20 May 2016; Revised 28 November 2016; Accepted 21 December 2016; Published 9 March 2017

Academic Editor: Filippo Ubertini

Copyright ( 2017 Jie Sun and Jing Zhang. This is an open access article distributed under the Creative Commons Attribution License, which permits unrestricted use, distribution, and reproduction in any medium, provided the original work is properly cited.

In this study, we focus on stability analysis for systems with time-varying delay and nonlinear perturbations. In order to cut down the conservatism of the existing stability criteria, we utilize the triple integral forms of Lyapunov-Krasovskii functional (LKF). In addition, by using single and double integral forms of Wirtinger-based inequality, we overcome some conservatism which come from Jensen's inequality. Three well-known numerical examples are given at the end. Compared with some existing results, our results have less conservatism.

\section{Introduction}

Time-delay has attracted a lot of interests because it is widely encountered in communication systems, neural networks, economic systems, biological systems, and networked control systems [1-6]. Since time-delay will cause serious degradation of system performance, a lot of researchers are making considerable effort for stability analysis of time-delay systems in the last few decades. The LKF approach and LMI method are the efficient instrument to get the delay-dependent stability criterion. However, only a sufficient condition we can get by utilizing these methods. Therefore, we focus on cutting down the conservatism of stability criterion. As we know, the maximum allowable delay bound (MADB) of the time-delay can measure the conservatism of stability criterion. We can get the larger MADB of time-varying delay according to the stability criterion with less conservatism.

In a lot of existing results, Jensen's inequality has played an important role. However, it will induce some conservatism hard to overcome. To cut down the conservatism, Wirtingerbased integral inequality [7], which can be used to obtain much tighter lower bound of single integral terms, was proposed. Very recently, based on Wirtinger-based integral inequality, a Wirtinger-based double integral inequality was proposed to get much tighter lower bound of double integral terms [8].

In a lot of recent literatures [9-11], researchers only utilize single and double integral forms of LKF to derive delaydependent stability criterion. We believe that triple integral form of LKF is helpful for improving the performance of former criteria.

Motivated by the previous discussions, we are concerned about the stability analysis for the time-varying delay systems with nonlinear perturbations. We introduce the triple integral forms of LKF to cut down the conservatism. Taking the time derivative of $\int_{t-h}^{t} \int_{s}^{t} \int_{u}^{t} \dot{x}^{T}(v) R_{i} \dot{x}(v) d v d u d s$, we obtain $-\int_{t-h}^{t} \int_{s}^{t} \dot{x}^{T}(u) R_{i} \dot{x}(u) d u d s$. Instead of Jensen's inequality, Wirtinger-based double integral inequality was utilized to find much tighter upper bound of $-\int_{a}^{b} \int_{u}^{b} \dot{x}^{T}(u) R_{i} \dot{x}(u) d u d s$. Compared with some existing results, our results have less conservatism.

The organization of this paper is as follows. The system is presented in Section 2, and some useful lemmas are given. Then the new stability criterion and proof are given in Section 3. In Section 4, the effectiveness of our results can be illustrated by three examples. Section 5 is the conclusion of our investigation. 
Notations. In this paper, the superscript $T$ means the transpose of a matrix; $\operatorname{col}\{\cdot\}$ denotes the column vector.

\section{Problem Formulation and Preliminaries}

The time-varying delay systems with nonlinear perturbations are considered as follows:

$$
\begin{aligned}
& \dot{x}(t)=A x(t)+B x(t-\tau(t))+\vartheta(t), \\
& \vartheta(t)=h(x(t), t)+p(x(t-\tau(t)), t), \\
& x(t)=\phi(t), \quad t \in\left[-d_{M}, 0\right]
\end{aligned}
$$

with $x(t) \in \mathbb{R}^{n}$ as the state variate; $A, B \in \mathbb{R}^{n * n}$ are predefined constant matrices; $d(t) \geq 0$ represent a time-varying delay satisfying

$$
d(t) \in\left[d_{m}, d_{M}\right], \quad \dot{d}(t) \leq d_{d}
$$

The nonlinear perturbations $h(x(t), t) \in \mathbb{R}^{n}$ and $p(x(t-$ $d(t)), t) \in \mathbb{R}^{n}$ can be abbreviated as $h$ and $p$, assumed as follows:

$$
\begin{aligned}
& h^{T} h \leq \alpha^{2} x^{T}(t) C^{T} C x(t), \\
& p^{T} p \leq \beta^{2} x^{T}(t-d(t)) D^{T} D x(t-d(t)),
\end{aligned}
$$

where $\alpha$ and $\beta$ are positive scalars and $C, D \in \mathbb{R}^{n * n}$ are constant matrices.

In order to derive improved stability criterion, the following lemmas will be used.

Lemma 1 (see [7]). $Z$ is a symmetric positive definite matrix, for differentiable function $x \in\left[d_{1}, d_{2}\right] \rightarrow \mathbb{R}^{n}$, and we can obtain

$$
\int_{d_{1}}^{d_{2}} \dot{x}^{T}(s) Z \dot{x}(s) d s \geq \frac{1}{d_{2}-d_{1}}\left[\begin{array}{l}
\varepsilon_{1} \\
\varepsilon_{2}
\end{array}\right]^{T}\left[\begin{array}{ll}
Z & 0 \\
* & Z
\end{array}\right]\left[\begin{array}{l}
\varepsilon_{1} \\
\varepsilon_{2}
\end{array}\right],
$$

where

$$
\begin{aligned}
& \varepsilon_{1}=x\left(d_{1}\right)-x\left(d_{2}\right) \\
& \varepsilon_{2}=\sqrt{3} x\left(d_{1}\right)+\sqrt{3} x\left(d_{2}\right)-\frac{2 \sqrt{3}}{d_{2}-d_{1}} \int_{d_{1}}^{d_{2}} x(s) d s .
\end{aligned}
$$

Lemma 2 (see [28]). For positive definite $d_{v}: \mathbb{R}^{n} \rightarrow \mathbb{R}, d_{v} \in$ $\mathscr{G} \subseteq \mathbb{R}^{n}(v=1 \cdots N)$, reciprocally convex combination of $d_{v}$ can be written as

$$
\begin{aligned}
\min _{\left\{\rho_{m} \mid \rho_{m}>0, \sum_{m} \rho_{m}=1\right\}} & \sum_{m} \frac{1}{\rho_{m}} d_{m}(t)=\sum_{m} d_{m}(t)+\max _{l_{m, w}(t)} \sum_{m \neq w} l_{m, w}(t) \\
\text { subject to } & \left\{l_{m, w}: \mathbb{R}^{n} \longrightarrow \mathbb{R}, l_{w, m}(t) \triangleq l_{m, w}(t),\left[\begin{array}{cc}
d_{m}(t) & l_{m, w}(t) \\
l_{m, w}(t) & d_{w}(t)
\end{array}\right] \geq 0\right\} .
\end{aligned}
$$

Lemma 3 (see [8]). $R$ is a symmetric positive definite matrix, for differentiable function $x \in\left[h_{a}, h_{b}\right] \rightarrow \mathbb{R}^{n}$, and we have

$$
\begin{gathered}
\frac{\left(h_{b}-h_{a}\right)^{2}}{2} \int_{h_{a}}^{h_{b}} \int_{s}^{h_{b}} \dot{x}^{T}(u) R \dot{x}(u) d u d s \\
\geq\left[\begin{array}{l}
\omega_{1} \\
\omega_{2}
\end{array}\right]^{T}\left[\begin{array}{ll}
R & 0 \\
* & R
\end{array}\right]\left[\begin{array}{l}
\omega_{1} \\
\omega_{2}
\end{array}\right],
\end{gathered}
$$

where

$$
\begin{aligned}
& \omega_{1}=\left(h_{b}-h_{a}\right) x\left(h_{b}\right)-\int_{h_{a}}^{h_{b}} x(s) d s, \\
& \omega_{2}=\frac{\sqrt{2}\left(h_{b}-h_{a}\right)}{2} x\left(h_{b}\right)+\sqrt{2} \int_{h_{a}}^{h_{b}} x(s) d s
\end{aligned}
$$

$$
-\frac{3 \sqrt{2}}{h_{b}-h_{a}} \int_{h_{a}}^{h_{b}} \int_{s}^{h_{b}} x(u) d u d s
$$

\section{Stability Criterion}

Theorem 4. System (1) satisfying (2)-(3) will be asymptotically stable if there exist given scalars $d_{m}, d_{M}$, and $d_{d}$ and $\epsilon_{1}>0$ and $\epsilon_{2}>0$ and positive symmetric matrices $P \in \mathbb{R}^{5 n * 5 n}$, $W_{s} \in \mathbb{R}^{n * n}(s=1,2,3)$, and $Z_{q} \in \mathbb{R}^{n * n}(q=1, \ldots, 4)$ and appropriate dimensions matrices $S_{m}$, satisfying the following LMIs:

$$
\begin{aligned}
& {\left[\begin{array}{cc}
\Phi & \Delta^{T} N \\
* & -N
\end{array}\right]<0,} \\
& {\left[\begin{array}{cc}
Z_{2} & S_{m} \\
* & Z_{2}
\end{array}\right] \geq 0, \quad(m=1,2),}
\end{aligned}
$$




$$
\begin{aligned}
& \Phi=G_{1}^{T} P G_{2}+G_{2}^{T} P G_{1}+Q+M_{1}+M_{2}-d_{m}^{2} \sum_{i=1}^{2} \Gamma_{i}^{T} Z_{1} \Gamma_{i}-\sum_{i=3}^{6} \Gamma_{i}^{T} Z_{2} \Gamma_{i}-\Gamma_{3}^{T} S_{1} \Gamma_{5}-\Gamma_{5}^{T} S_{1}^{T} \Gamma_{3}-\Gamma_{4}^{T} S_{2} \Gamma_{6}-\Gamma_{6}^{T} S_{2}^{T} \Gamma_{4} \\
& -d_{m}^{2} \sum_{i=7}^{8} \Gamma_{i}^{T} Z_{3} \Gamma_{i}-d_{M m}^{2} \sum_{i=9}^{10} \Gamma_{i}^{T} Z_{4} \Gamma_{i} \\
& G_{1}=\operatorname{col}\left\{e_{1}, e_{5}, e_{6}, e_{7}, e_{8}\right\} \text {, } \\
& G_{2}=\operatorname{col}\left\{A e_{1}+B e_{3}+e_{11}+e_{12}, e_{1}-e_{2}, e_{2}-e_{4}, d_{m} e_{1}-e_{5}, d_{M m} e_{2}-e_{6}\right\},
\end{aligned}
$$

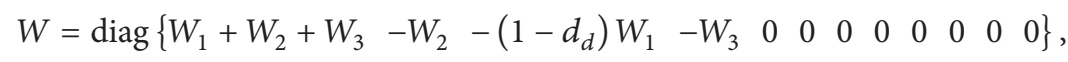

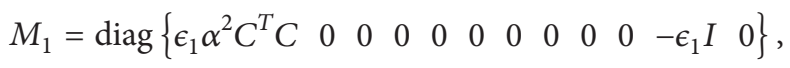

$$
\begin{aligned}
& M_{2}=\operatorname{diag}\left\{\begin{array}{llllllllllll}
0 & 0 & \epsilon_{2} \beta^{2} D^{T} D & 0 & 0 & 0 & 0 & 0 & 0 & 0 & 0 & \left.-\epsilon_{2} I\right\}
\end{array}\right. \\
& \Gamma_{1}=e_{1}-e_{2} \\
& \Gamma_{2}=\sqrt{3} e_{1}+\sqrt{3} e_{2}-\frac{2 \sqrt{3}}{d_{m}} e_{5} \\
& \Gamma_{3}=e_{2}-e_{3}, \\
& \Gamma_{4}=\sqrt{3} e_{2}+\sqrt{3} e_{3}-2 \sqrt{3} e_{9}, \\
& \Gamma_{5}=e_{3}-e_{4}, \\
& \Gamma_{6}=\sqrt{3} e_{3}+\sqrt{3} e_{4}-2 \sqrt{3} e_{10}, \\
& \Gamma_{7}=d_{m} e_{1}-e_{5}, \\
& \Gamma_{8}=\frac{\sqrt{2}}{2} d_{m} e_{1}+\sqrt{2} e_{5}-\frac{3 \sqrt{2}}{d_{m}} e_{7}, \\
& \Gamma_{9}=d_{M m} e_{2}-e_{6} \text {, } \\
& \Gamma_{10}=\frac{\sqrt{2} d_{M m}}{2} e_{2}+\sqrt{2} e_{6}-\frac{3 \sqrt{2}}{d_{M m}} e_{8} \\
& \Delta=A e_{1}+B e_{3}+e_{11}+e_{12}, \\
& d_{M m}=d_{M}-d_{m} \\
& e_{i}=\left[\begin{array}{lll}
0_{n \times(k-1) n} & I & 0_{n \times(12-k) n}
\end{array}\right], \quad k=1,2, \ldots, 12 \\
& N=N_{1}+N_{2}=d_{m}^{4} Z_{1}+d_{M m}^{2} Z_{2}+\frac{d_{m}^{6}}{4} Z_{3}+\frac{d_{M m}^{6}}{4} Z_{4} \text {. }
\end{aligned}
$$

Proof. Construct the following LKF:

$$
V(t)=\sum_{i=1}^{4} V_{i}(t)
$$

where

$$
V_{1}(t)=v^{T}(t) P v(t),
$$

$$
V_{2}(t)
$$$$
=\int_{t-d(t)}^{t} x^{T}(s) W_{1} x(s) d s+\int_{t-d_{m}}^{t} x^{T}(s) W_{2} x(s) d s
$$$$
+\int_{t-d_{\mathrm{M}}}^{t} x^{T}(s) W_{3} x(s) d s
$$ 
$V_{3}(t)$

$$
\begin{aligned}
= & d_{m}^{3} \int_{-d_{m}}^{0} \int_{t+s}^{t} \dot{x}^{T}(u) Z_{1} \dot{x}(u) d u d s \\
& +d_{M m} \int_{-d_{M}}^{-d_{m}} \int_{t+s}^{t} \dot{x}^{T}(u) Z_{2} \dot{x}(u) d u d s,
\end{aligned}
$$

$V_{4}(t)$

$$
\begin{aligned}
= & \frac{d_{m}^{4}}{2} \int_{t-d_{m}}^{t} \int_{s}^{t} \int_{u}^{t} \dot{x}^{T}(v) Z_{3} \dot{x}(v) d v d u d s \\
& +\frac{d_{M m}^{4}}{2} \int_{t-d_{M}}^{t-d_{m}} \int_{s}^{t-d_{m}} \int_{u}^{t} \dot{x}^{T}(v) Z_{4} \dot{x}(v) d v d u d s
\end{aligned}
$$$$
u_{1}(t)=\int_{t-d_{m}}^{t} x^{T}(s) d s \text {, }
$$$$
u_{2}(t)=\int_{t-d_{M}}^{t-d_{m}} x^{T}(s) d s,
$$$$
u_{3}(t)=\int_{t-d_{m}}^{t} \int_{s}^{t} x^{T}(u) d u d s,
$$$$
u_{4}(t)=\int_{t-d_{M}}^{t-d_{m}} \int_{s}^{t-d_{m}} x^{T}(u) d u d s,
$$$$
v^{T}(t)=\left[\begin{array}{lllll}
x^{T}(t) & u_{1}(t) & u_{2}(t) & u_{3}(t) & u_{4}(t)
\end{array}\right] .
$$

The time derivative of $V(t)$ is as follows:

$$
\dot{V}(t)=\sum_{i=1}^{4} \dot{V}_{i}(t),
$$

$$
\begin{aligned}
N_{1} & =d_{m}^{4} Z_{1}+d_{M m}^{2} Z_{2}, \\
N_{2} & =\frac{d_{m}^{6}}{4} Z_{3}+\frac{d_{M m}^{6}}{4} Z_{4}, \\
\zeta_{1}(t) & =-d_{m}^{3} \int_{t-d_{m}}^{t} \dot{x}^{T}(s) Z_{1} \dot{x}(s) d s, \\
\zeta_{2}(t) & =-d_{M m} \int_{t-d(t)}^{t-d_{m}} \dot{x}^{T}(s) Z_{2} \dot{x}(s) d s, \\
\zeta_{3}(t) & =-d_{M m} \int_{t-d_{M}}^{t-d(t)} \dot{x}^{T}(s) Z_{2} \dot{x}(s) d s, \\
\zeta_{4}(t) & =-\frac{d_{m}^{4}}{2} \int_{t-d_{m}}^{t} \int_{s}^{t} \dot{x}^{T}(u) Z_{3} \dot{x}(u) d u d s, \\
\zeta_{5}(t) & =-\frac{d_{M m}^{4} \int_{t-d_{M}}^{t-d_{m}} \int_{s}^{t-d_{m}} \dot{x}^{T}(u) Z_{4} \dot{x}(u) d u d s,}{2} \int_{t-d(t)}^{t-d_{m}} x^{T}(s) d s, \\
u_{5}(t) & =\frac{1}{d(t)-d_{m}}(t)=\frac{1}{d_{M}-d(t)} \int_{t-d_{M}}^{t-d(t)} x^{T}(s) d s, \\
\xi^{T}(t) & =\left[x^{T}(t) x^{T}\left(t-d_{m}\right) x^{T}(t-d(t)) x^{T}\left(t-d_{M}\right) u_{1}(t) \cdots u_{6}(t) h^{T} p^{T}\right] .
\end{aligned}
$$

$$
\begin{aligned}
& \dot{V}_{1}(t)=2 v^{T}(t) P \dot{\nu}(t)=\xi^{T}(t)\left(G_{1}^{T} P G_{2}+G_{2}^{T} P G_{1}\right) \xi(t) \\
& \dot{V}_{2}(t)=\sum_{i=1}^{3} x^{T}(t) W_{i} x(t)-(1-\dot{d}(t)) x^{T}(t-d(t)) \\
& * W_{1} x(t-d(t))-x^{T}\left(t-d_{m}\right) W_{2} x\left(t-d_{m}\right) \\
& -x^{T}\left(t-d_{M}\right) W_{3} x\left(t-d_{M}\right) \leq \xi^{T}(t) Q \xi(t), \\
& \dot{V}_{3}(t)=\dot{x}^{T}(t)\left[d_{m}^{4} Z_{1}+d_{M m}^{2} Z_{2}\right] \dot{x}(t)+\sum_{i=1}^{3} \zeta_{i}=\xi^{T}(t) \\
& \cdot \Delta^{T} N_{1} \Delta \xi(t)+\sum_{i=1}^{3} \zeta_{i}, \\
& \dot{V}_{4}(t)=\frac{d_{m}^{4}}{2} \int_{t-d_{m}}^{t} \int_{s}^{t}\left[\dot{x}^{T}(t) Z_{3} \dot{x}(t)-\dot{x}^{T}(u) Z_{3}\right. \\
& \quad * \Delta^{T} N_{2} \Delta \xi(t)+\sum_{i=4}^{5} \zeta_{i}, \\
& \cdot \int_{t-d_{M}}^{t-d_{m}} \int_{s}^{t-d_{m}}\left[\dot{x}^{T}(t) * Z_{4} \dot{x}(t)\right. \\
& \left.\quad \dot{x}^{T}(u) Z_{4} \dot{x}(u)\right] d u d s=\xi_{M m}^{T}(t) \\
& 2
\end{aligned}
$$

where 
According to Lemma 1, we obtain

$$
\begin{aligned}
& \zeta_{1}(t) \leq-d_{m}^{2}\left[\begin{array}{l}
\varepsilon_{1}(t) \\
\varepsilon_{2}(t)
\end{array}\right]^{T}\left[\begin{array}{ll}
Z_{1} & 0 \\
* & Z_{1}
\end{array}\right]\left[\begin{array}{l}
\varepsilon_{1}(t) \\
\varepsilon_{2}(t)
\end{array}\right], \\
& \zeta_{2}(t) \leq-\frac{1}{\rho_{1}}\left[\begin{array}{l}
\varepsilon_{3}(t) \\
\varepsilon_{4}(t)
\end{array}\right]^{T}\left[\begin{array}{cc}
Z_{2} & 0 \\
* & Z_{2}
\end{array}\right]\left[\begin{array}{l}
\varepsilon_{3}(t) \\
\varepsilon_{4}(t)
\end{array}\right], \\
& \zeta_{3}(t) \leq-\frac{1}{\rho_{2}}\left[\begin{array}{l}
\varepsilon_{5}(t) \\
\varepsilon_{6}(t)
\end{array}\right]^{T}\left[\begin{array}{cc}
Z_{2} & 0 \\
* & Z_{2}
\end{array}\right]\left[\begin{array}{l}
\varepsilon_{5}(t) \\
\varepsilon_{6}(t)
\end{array}\right],
\end{aligned}
$$

where

$$
\begin{aligned}
\rho_{1} & =\frac{d(t)-d_{m}}{d_{M m}}, \\
\rho_{2} & =\frac{d_{M}-d(t)}{d_{M m}}, \\
\varepsilon_{1}(t) & =x(t)-x\left(t-d_{m}\right), \\
\varepsilon_{2}(t) & =\sqrt{3} x(t)+\sqrt{3} x\left(t-d_{m}\right)-\frac{2 \sqrt{3}}{d_{m}} u_{1}(t), \\
\varepsilon_{3}(t) & =x\left(t-d_{m}\right)-x(t-d(t)), \\
\varepsilon_{4}(t) & =\sqrt{3} x\left(t-d_{m}\right)+\sqrt{3} x(t-d(t))-2 \sqrt{3} u_{5}(t), \\
\varepsilon_{5}(t) & =x(t-d(t))-x\left(t-d_{M}\right), \\
\varepsilon_{6}(t) & =\sqrt{3} x(t-d(t))+\sqrt{3} x\left(t-d_{M}\right)-2 \sqrt{3} u_{6}(t)
\end{aligned}
$$

and inequality (16) can be denoted as

$$
\zeta_{1}(t) \leq-\xi^{T}(t) d_{m}^{2}\left[\Gamma_{1}^{T} Z_{1} \Gamma_{1}+\Gamma_{2}^{T} Z_{1} \Gamma_{2}\right] \xi(t) .
$$

It is clear that the real numbers $\rho_{1}$ and $\rho_{2}$ satisfy $\rho_{1}>0, \rho_{2}>$ 0 , and $\rho_{1}+\rho_{2}=1$. Then introduce appropriate dimensions matrices $S_{1}$ and $S_{2}$, such that

$$
\begin{aligned}
& {\left[\begin{array}{cc}
Z_{2} & S_{2} \\
* & Z_{2}
\end{array}\right] \geq 0,} \\
& {\left[\begin{array}{cc}
Z_{2} & S_{1} \\
* & Z_{2}
\end{array}\right] \geq 0 .}
\end{aligned}
$$

Applying Lemma 2 to (17) and (18)

$$
\begin{aligned}
\zeta_{2}(t) & +\zeta_{3}(t) \leq-\left(\frac{1}{\rho_{1}} \varepsilon_{3}^{T}(t) Z_{2} \varepsilon_{3}(t)\right. \\
+ & \left.\frac{1}{\rho_{2}} \varepsilon_{5}^{T}(t) Z_{2} \varepsilon_{5}(t)\right)-\left(\frac{1}{\rho_{1}} \varepsilon_{4}^{T}(t) Z_{2} \varepsilon_{4}(t)\right. \\
+ & \left.\frac{1}{\rho_{2}} \varepsilon_{6}^{T}(t) Z_{2} \varepsilon_{6}(t)\right) \leq-\left[\begin{array}{l}
\varepsilon_{3}(t) \\
\varepsilon_{5}(t)
\end{array}\right]^{T} \\
\cdot & {\left[\begin{array}{ll}
Z_{2} & S_{1} \\
* & Z_{2}
\end{array}\right]\left[\begin{array}{l}
\varepsilon_{3}(t) \\
\varepsilon_{5}(t)
\end{array}\right]-\left[\begin{array}{l}
\varepsilon_{4}(t) \\
\varepsilon_{6}(t)
\end{array}\right]^{T}\left[\begin{array}{ll}
Z_{2} & S_{2} \\
* & Z_{2}
\end{array}\right]\left[\begin{array}{l}
\varepsilon_{4}(t) \\
\varepsilon_{6}(t)
\end{array}\right] }
\end{aligned}
$$

$$
\begin{aligned}
& =-\xi^{T}(t)\left[\sum_{i=3}^{6} \Gamma_{i}^{T} Z_{2} \Gamma_{i}+\Gamma_{3}^{T} S_{1} \Gamma_{5}+\Gamma_{5}^{T} S_{1}^{T} \Gamma_{3}+\Gamma_{4}^{T} S_{2} \Gamma_{6}\right. \\
& \left.+\Gamma_{6}^{T} S_{2}^{T} \Gamma_{4}\right] \xi(t) .
\end{aligned}
$$

Using Lemma 3 can lead to

$$
\begin{aligned}
\zeta_{4}(t) & \leq-d_{m}^{2}\left[\begin{array}{l}
\omega_{1}(t) \\
\omega_{2}(t)
\end{array}\right]^{T}\left[\begin{array}{ll}
Z_{3} & 0 \\
* & Z_{3}
\end{array}\right]\left[\begin{array}{l}
\omega_{1}(t) \\
\omega_{2}(t)
\end{array}\right] \\
& =-\xi^{T}(t) d_{m}^{2} \sum_{i=7}^{8} \Gamma_{i}^{T} Z_{3} \Gamma_{i} \xi(t), \\
\zeta_{5}(t) & \leq-d_{M m}^{2}\left[\begin{array}{l}
\omega_{3}(t) \\
\omega_{4}(t)
\end{array}\right]^{T}\left[\begin{array}{cc}
Z_{4} & 0 \\
* & Z_{4}
\end{array}\right]\left[\begin{array}{l}
\omega_{3}(t) \\
\omega_{4}(t)
\end{array}\right] \\
& =-\xi^{T}(t) d_{M m}^{2} \sum_{i=9}^{10} \Gamma_{i}^{T} Z_{4} \Gamma_{i} \xi(t),
\end{aligned}
$$

where

$$
\begin{aligned}
& \omega_{1}(t)=d_{m} x(t)-u_{1}(t), \\
& \omega_{2}(t)=\frac{\sqrt{2} d_{m}}{2} x(t)+\sqrt{2} u_{1}(t)-\frac{3 \sqrt{2}}{d_{m}} u_{3}(t), \\
& \omega_{3}(t)=d_{M m} x\left(t-d_{m}\right)-u_{2}(t), \\
& \omega_{4}(t)=\frac{\sqrt{2} d_{M m}}{2} x\left(t-d_{m}\right)+\sqrt{2} u_{2}(t)-\frac{3 \sqrt{2}}{d_{M m}} u_{4}(t) .
\end{aligned}
$$

Form (3), we have

$$
\begin{aligned}
\epsilon_{1}\left(h^{T} h-\alpha^{2} x^{T}(t) C^{T} C x(t)\right) & \leq 0, \\
\epsilon_{2}\left(p^{T} p-\beta^{2} x^{T}(t-d(t)) D^{T} D x(t-d(t))\right) & \leq 0 .
\end{aligned}
$$

Combining (13), (14), (20), (22), (23), and (25), we obtain

$$
\begin{gathered}
\dot{V}(t) \leq \xi^{T}(t)\left[G_{1}^{T} P G_{2}+G_{2}^{T} P G_{1}+W+M_{1}+M_{2}\right. \\
+\Delta^{T} N \Delta-d_{m}^{2} \sum_{i=1}^{2} \Gamma_{i}^{T} Z_{1} \Gamma_{i}-\sum_{i=3}^{6} \Gamma_{i}^{T} Z_{2} \Gamma_{i}-\Gamma_{3}^{T} S_{1} \Gamma_{5} \\
-\Gamma_{5}^{T} S_{1}^{T} \Gamma_{3}-\Gamma_{4}^{T} S_{2} \Gamma_{6}-\Gamma_{6}^{T} S_{2}^{T} \Gamma_{4}-d_{m}^{2} \sum_{i=7}^{8} \Gamma_{i}^{T} Z_{3} \Gamma_{i} \\
\left.\quad-d_{M m}^{2} \sum_{i=9}^{10} \Gamma_{i}^{T} Z_{4} \Gamma_{i}\right] \xi(t) .
\end{gathered}
$$

Using Schur complement, (26) can be transform to the first LMI of (9), which completed the proof of Theorem 4. 
When $h=p=0$, that means system without perturbation. We can consider the following system:

$$
\begin{aligned}
& \dot{x}(t)=A x(t)+B x(t-d(t)), \\
& x(t)=\phi(t), \quad t \in\left[-d_{M}, 0\right] .
\end{aligned}
$$

Stability analysis of system (26) is studied in a lot of literatures. From Theorem 4, the stability criterion of system (26) can be obtained easily.
Corollary 5. System (26) under condition (2) will be asymptotically stable if there exist given scalars $d_{m}, d_{M}$, and $d_{d}$ and positive symmetric matrices $P \in \mathbb{R}^{5 n * 5 n}, W_{s} \in \mathbb{R}^{n * n}(s=$ $1,2,3)$, and $Z_{q} \in \mathbb{R}^{n * n}(q=1, \ldots, 4)$ and appropriate dimensions matrices $S_{m}$, satisfying the following LMIs:

$$
\begin{aligned}
& {\left[\begin{array}{cc}
\Phi & \Delta^{T} N \\
* & -N
\end{array}\right]<0,} \\
& {\left[\begin{array}{cc}
Z_{2} & S_{m} \\
* & Z_{2}
\end{array}\right] \geq 0, \quad(m=1,2),}
\end{aligned}
$$

where

$$
\begin{aligned}
\Phi & =G_{1}^{T} P G_{2}+G_{2}^{T} P G_{1}+W-d_{m}^{2} \sum_{i=1}^{2} \Gamma_{i}^{T} Z_{1} \Gamma_{i}-\sum_{i=3}^{6} \Gamma_{i}^{T} Z_{2} \Gamma_{i}-\Gamma_{3}^{T} S_{1} \Gamma_{5}-\Gamma_{5}^{T} S_{1}^{T} \Gamma_{3}-\Gamma_{4}^{T} S_{2} \Gamma_{6}-\Gamma_{6}^{T} S_{2}^{T} \Gamma_{4}-d_{m}^{2} \sum_{i=7}^{8} \Gamma_{i}^{T} Z_{3} \Gamma_{i} \\
& -d_{M m}^{2} \sum_{i=9}^{10} \Gamma_{i}^{T} Z_{4} \Gamma_{i}, \\
G_{1} & =\operatorname{col}\left\{e_{1}, e_{5}, e_{6}, e_{7}, e_{8}\right\} \\
G_{2} & =\operatorname{col}\left\{A e_{1}+B e_{3}, e_{1}-e_{2}, e_{2}-e_{4}, d_{m} e_{1}-e_{5}, d_{M m} e_{2}-e_{6}\right\}, \\
W & =\operatorname{diag}\left\{W_{1}+W_{2}+W_{3}-W_{2}-\left(1-d_{d}\right) W_{1}-W_{3} \quad 0 \quad 0 \quad 0 \quad 0 \quad 0 \quad 0\right\}, \\
\Delta & =A e_{1}+B e_{3}, \\
e_{i} & =\left[\begin{array}{llll}
0_{n \times(k-1) n} I & 0_{n \times(10-k) n}
\end{array}\right],
\end{aligned}
$$

$\Gamma_{1} \cdots \Gamma_{10}$ and $N$ are defined in Theorem 4. If the information of delay is unavailable, by setting $Q_{1}=0$, we obtain

Corollary 6. If there exist given scalars $d_{m}, d_{M}$, and $d_{d}$ and positive symmetric matrices $P \in \mathbb{R}^{5 n * 5 n}, W_{s} \in \mathbb{R}^{n * n}(s=$ $1,2,3)$, and $Z_{q} \in \mathbb{R}^{n * n}(q=1, \ldots, 4)$ and appropriate dimensions matrices $S_{m}$, such that (27) with $W_{1}=0$ are feasible, system (26) under condition (2) will be asymptotically stable.

Remark 7. From the viewpoint of control theory, the changed trend of system in reality is not only decided by the current states but also related to its past states. This is why the study of time-delay system is significant. This paper is interested in the stability analysis of the system with time-delay which has strong background in reality. The stability criterion we proposed can be used to design effective control strategy in a specific engineering file, such as synchronization of coronary artery. In order to compare with exiting results, a lot of papers related to stability analysis are concerned about the MADB which can be calculated by stability criterion. We can see the same particular examples in [10-30] as Examples 1-3 in our manuscript. In these examples structure of the matrices is prescribed. The purpose of these examples is to measure the conservatism of proposed criterion. The larger MADB, the less conservatism.

\section{Numerical Examples}

The advantages of our results can be illustrated by the following examples.

Example 1. Nonlinear system (1) is subject to (2) and (3) with

$$
\begin{aligned}
& A=\left[\begin{array}{cc}
-1.2 & 0.1 \\
-0.1 & -1
\end{array}\right], \\
& B=\left[\begin{array}{cc}
-0.6 & 0.7 \\
-1 & -0.8
\end{array}\right], \\
& C=D=\left[\begin{array}{ll}
1 & 0 \\
0 & 1
\end{array}\right] .
\end{aligned}
$$

For given $d_{m}, \alpha, \beta$, and $d_{d}$, utilizing Theorem 4 , we calculate the MADB $d_{M}$ which can guarantee the stability of system (1). The result is listed in Table 1 . We can see that the stability criterion we proposed has less conservatism than others. It is worth mentioning that when $\alpha=0$, Theorem 4 
TABLE 1: Maximal $d_{M}$ for given $d_{m}=0.5$.

\begin{tabular}{|c|c|c|c|c|c|c|}
\hline \multirow{3}{*}{ Methods } & \multicolumn{6}{|c|}{$\alpha, \beta$} \\
\hline & \multicolumn{3}{|c|}{$\alpha=0, \beta=0.1$} & \multicolumn{3}{|c|}{$\alpha=0.1, \beta=0.1$} \\
\hline & $d_{d}=0.5$ & $d_{d}=0.9$ & $d_{d}=1.1$ & $d_{d}=0.5$ & $d_{d}=0.9$ & $d_{d}=1.1$ \\
\hline [12] & 1.4420 & 1.3380 & 1.3380 & 1.2840 & 1.2450 & 1.2450 \\
\hline$[10](N=2)$ & 1.5500 & 1.5500 & 1.5500 & 1.3690 & 1.3690 & 1.3690 \\
\hline [13] & 1.5580 & 1.5580 & 1.5580 & 1.3840 & 1.3840 & 1.3840 \\
\hline [14] & 1.5636 & 1.5636 & 1.5636 & 1.3858 & 1.3858 & 1.3858 \\
\hline$[10](N=4)$ & 1.8240 & 1.8240 & 1.8240 & 1.5240 & 1.5240 & 1.5240 \\
\hline [15] & 1.8599 & 1.8599 & 1.8599 & 1.6622 & 1.6622 & 1.6622 \\
\hline Theorem 4 & 2.2561 & 2.2561 & 2.2561 & 1.6844 & 1.6844 & 1.6844 \\
\hline
\end{tabular}

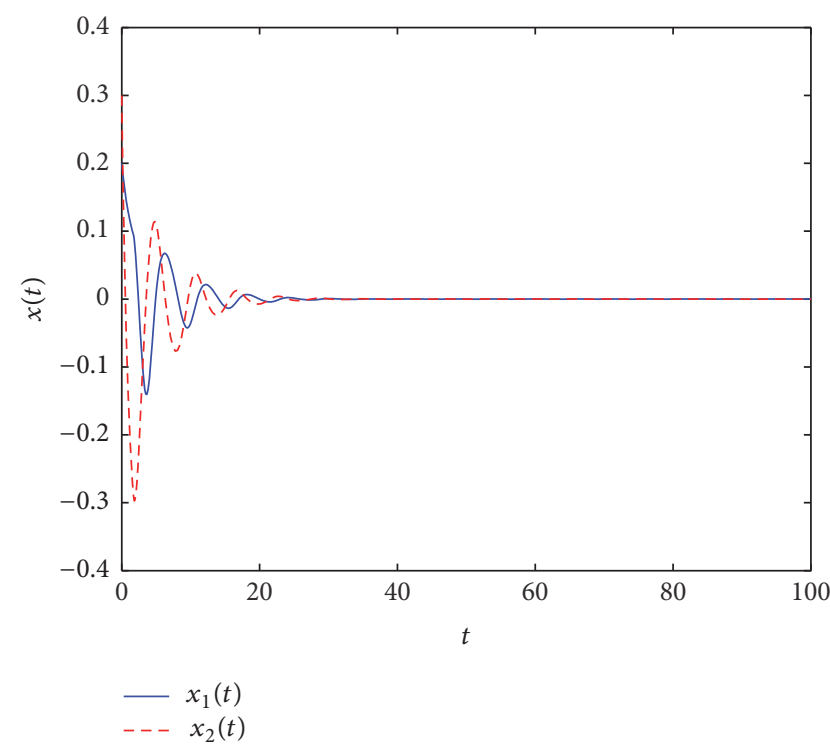

(a) $d(t)=1.0922+0.5922 * \sin (1.5 t)$

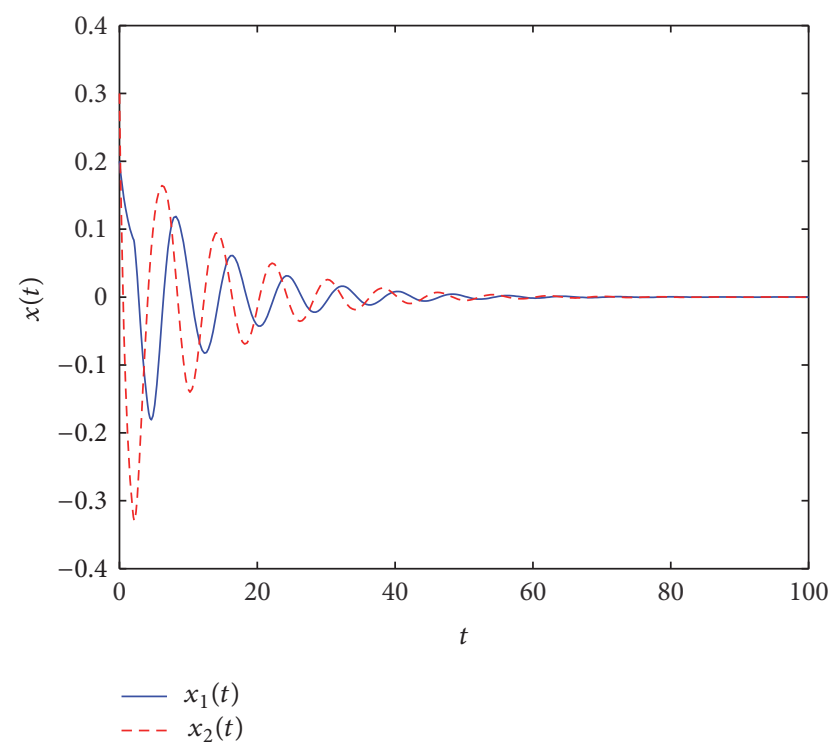

(b) $d(t)=2.0497$

FIGURE 1: Trajectories of $x(t)$.

TABLE 2: Maximal $d_{M}$ with given $d_{m}=0$ for $d_{d}=0$.

\begin{tabular}{lcc}
\hline Methods & \multicolumn{2}{c}{$\alpha, \beta$} \\
& $\alpha=0, \beta=0.1$ & $\alpha=0.1, \beta=0.1$ \\
\hline$[16]$ & 0.6811 & 0.6129 \\
{$[17]$} & 1.3279 & 1.2503 \\
{$[18]$} & 2.7422 & 1.8753 \\
{$[19]$} & 2.7423 & 1.8753 \\
{$[20]$} & 2.7757 & 1.8959 \\
{$[21]$} & 2.7758 & 1.8959 \\
{$[22]$} & 2.9816 & 1.9805 \\
Theorem 4 & 3.0715 & 2.0497 \\
\hline
\end{tabular}

can get much better results. Then we consider system (1) with constant time-delay. When $d_{d}=0$, the MADB $d_{M}$ under different $\alpha$ and $\beta$ is listed in Table 2. We can see that Theorem 4 provides much less conservative results than others.

Let $f(x(t))=0.1 x(t) * \sin (x(t)), g(x(t-d(t)))=0.1 x(t-$ $d(t)) * \cos (x(t-d(t)))$, and $x(0)=(0.2,0.3)$. Figure 1 (a) shows the trajectories of variable $x(t)$ with $\alpha=0.1, \beta=0.1, d_{d}=0.5$, $d_{m}=0.5$, and $d(t)=1.0922+0.5922 * \sin (1.5 t)$. Figure $1(\mathrm{~b})$ shows the trajectories of variable $x(t)$ with $\alpha=0.1, \beta=0.1$, $d_{d}=0, d_{m}=0$, and $d(t)=2.0497$.

Example 2. Consider system (26) with

$$
\begin{aligned}
& A=\left[\begin{array}{cc}
-2 & 0 \\
0 & -0.9
\end{array}\right], \\
& B=\left[\begin{array}{cc}
-1 & 0 \\
-1 & -1
\end{array}\right] .
\end{aligned}
$$

For given $d_{m}=3,4,5$ and different $d_{d}=0.1,0.3,0.5,0.9$, we calculate the MADB $d_{M}$ which is listed in Table 3 . Table 3 illustrates the methods presented in Corollary 5 providing less conservative results than others. It is worth noting that the effect is very obvious when $d_{d} \leq 0.5$.

Let $d(t)=5.1964+0.1964 * \sin (4.58 * t)$, and trajectories of variable $x(t)$ can be showed in Figure 2 . 
TABLE 3: Maximal $d_{M}$ with given $d_{m}$ for different $d_{d}$.

\begin{tabular}{|c|c|c|c|c|c|}
\hline$d_{m}$ & Methods & $d_{d}=0.1$ & $d_{d}=0.3$ & $d_{d}=0.5$ & $d_{d}=0.9$ \\
\hline \multirow{4}{*}{3} & [23] & 4.3979 & 3.3408 & 3.3408 & 3.3408 \\
\hline & {$[24]$} & 4.4506 & 3.4186 & 3.4186 & 3.4186 \\
\hline & [11] & 4.8247 & 3.6616 & 3.6616 & 3.6616 \\
\hline & Corollary 5 & 5.1213 & 4.2496 & 4.2208 & 3.5974 \\
\hline \multirow{4}{*}{4} & {$[23]$} & 4.1978 & 4.1690 & 4.1690 & 4.1690 \\
\hline & {$[24]$} & 4.2367 & 4.2097 & 4.2097 & 4.2097 \\
\hline & [11] & 4.5762 & 4.3788 & 4.3788 & 4.3788 \\
\hline & Corollary 5 & 5.1916 & 4.9391 & 4.9338 & 4.4987 \\
\hline \multirow{4}{*}{5} & {$[23]$} & 5.0275 & 5.0275 & 5.0275 & 5.0275 \\
\hline & {$[24]$} & 5.0440 & 5.0440 & 5.0440 & 5.0440 \\
\hline & [11] & 5.1453 & 5.1453 & 5.1453 & 5.1453 \\
\hline & Corollary 5 & 5.7065 & 5.7043 & 5.6965 & 5.3928 \\
\hline
\end{tabular}

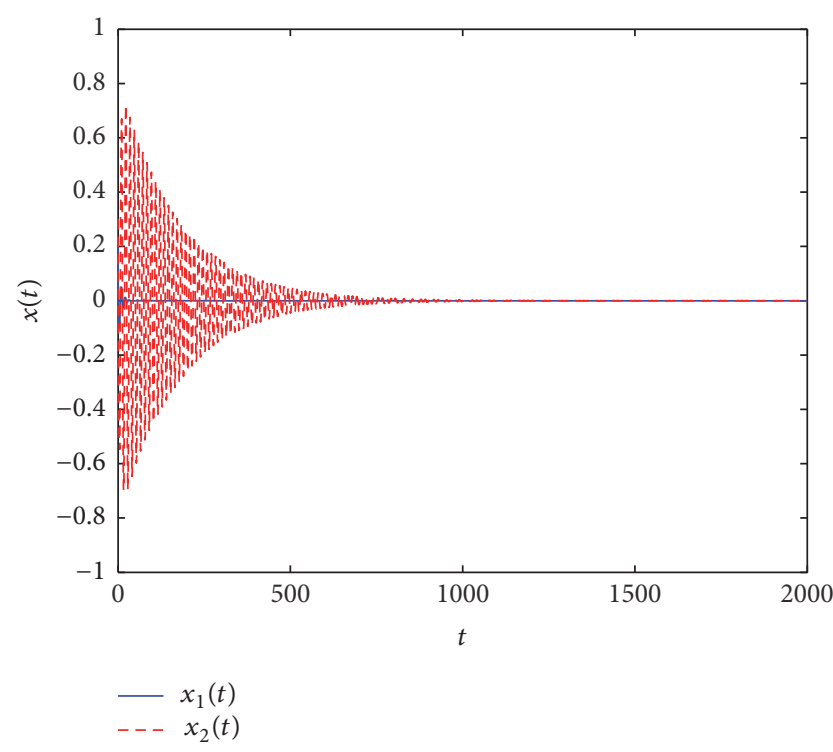

FIGURE 2: Trajectories of $x(t)$ with $d(t)=5.1964+0.1964 * \sin (4.58 *$ t).

Example 3. Consider another system (26) with

$$
\begin{aligned}
& A=\left[\begin{array}{cc}
0 & 1 \\
-1 & -2
\end{array}\right], \\
& B=\left[\begin{array}{cc}
0 & 0 \\
-1 & 1
\end{array}\right] .
\end{aligned}
$$

Sometimes the information of $d(t)$ is unavailable or $d(t)$ is undifferentiable. We calculate the MADB $d_{M}$ under different $d_{m}$ for unknown $d_{d}$. From Table 4 , it is observed that the results obtained by Corollary 6 are much less conservative than others. Finally, for given $d_{m}=0$ and different $d_{d}=$ $0.1,0.3$, using Corollary 5 we get the MADB $d_{M}$ listed in Table 5. It is clear to see that our results have much less conservatism when the lower bound is $d_{m}=0$.

Let $d(t)=1.54685+1.54685 * \sin (0.194 * t)$, and trajectories of variable $x(t)$ can be showed in Figure 3 .
TABLE 4: Maximal $d_{M}$ with given $d_{m}$ for unknown $d_{d}$.

\begin{tabular}{lccc}
\hline Methods & $d_{m}=0.3$ & $d_{m}=0.5$ & $d_{m}=0.8$ \\
\hline$[25]$ & 1.0715 & 1.2191 & 1.4539 \\
{$[23](N=2)$} & 1.0716 & 1.2196 & 1.4552 \\
{$[26]$} & 1.2043 & 1.3429 & 1.5663 \\
{$[27]$} & 1.2246 & 1.3619 & 1.5838 \\
{$[28]$} & 1.2400 & 1.3800 & 1.6000 \\
{$[29]$} & 1.2700 & 1.3900 & 1.6100 \\
{$[30]$} & 1.3500 & 1.4700 & 1.6800 \\
Corollary 6 & 1.5607 & 1.6426 & 1.7101 \\
\hline
\end{tabular}

TABLE 5: Maximal $d_{M}$ with given $d_{m}=0$ for $d_{d}=0.1,0.3$.

\begin{tabular}{lcc}
\hline Methods & $d_{d}=0.1$ & $d_{d}=0.3$ \\
\hline$[25]$ & 5.4630 & 2.2160 \\
{$[23](N=2)$} & 5.4764 & 2.2160 \\
{$[26]$} & 5.4780 & 2.2850 \\
{$[27]$} & 5.4940 & 2.3070 \\
Corollary 5 & 7.6301 & 3.0937 \\
\hline
\end{tabular}

\section{Conclusion}

We have researched the stability analysis problem for the time-varying delay systems. We utilize the new augmented LKF which is constructed based on single and double integral forms of Wirtinger-based inequality. Combining reciprocally convex method, we get an improved delay-dependent stability criterion. Finally, examples illustrate that the stability criterion we obtained is less conservative than some existing results.

\section{Competing Interests}

The authors declare that there is no conflict of interests regarding the publication of this paper. 


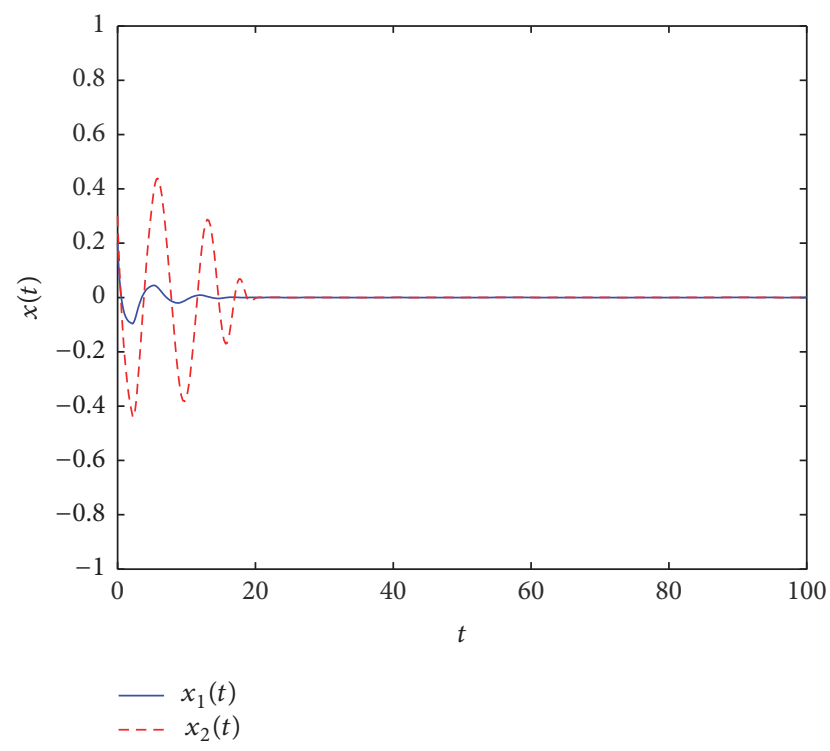

FIGURE 3: Trajectories of $x(t)$ with $d(t)=1.54685+1.54685 *$ $\sin (0.194 * t)$.

\section{Acknowledgments}

This work is supported by the Science and Technology Commission of Tianjin Municipality (Grant no. 15JCYBJC16100).

\section{References}

[1] H. Gao, T. Chen, and J. Lam, "A new delay system approach to network-based control," Automatica, vol. 44, no. 1, pp. 39-52, 2008.

[2] J.-L. Wang, H.-N. Wu, and T. Huang, "Passivity-based synchronization of a class of complex dynamical networks with timevarying delay," Automatica. A Journal of IFAC, the International Federation of Automatic Control, vol. 56, pp. 105-112, 2015.

[3] X. Jiang, Q. L. Han, S. Liu, and A. Xue, "A new H1 stabilization criterion for networked control systems," IEEE Transactions on Automatic Control, vol. 53, pp. 1025-1032, 2008.

[4] H. Y. Li, C. W. Wu, P. Shi, and Y. B. Gao, "Control of nonlinear networked systems with packet dropouts: interval type-2 fuzzy model-based approach," IEEE Transactions on Cybernetics, vol. 45, no. 11, pp. 2378-2389, 2015.

[5] H. Y. Li, C. W. Wu, L. G. Wu, H. K. Lam, and Y. B. Gao, "Filtering of interval type-2 fuzzy systems with intermittent measurements," IEEE Transactions on Cybernetics, vol. 46, no. 3, pp. 668-678, 2016.

[6] T. Wang, H. Gao, and J. Qiu, "A combined adaptive neural network and nonlinear model predictive control for multirate networked industrial process control," IEEE Transactions on Neural Networks and Learning Systems, vol. 27, no. 2, pp. 416425, 2016.

[7] A. Seuret and F. Gouaisbaut, "Wirtinger-based integral inequality: application to time-delay systems," Automatica, vol. 49, no. 9, pp. 2860-2866, 2013.

[8] M. Park, O. Kwon, J. H. Park, S. Lee, and E. Cha, "Stability of time-delay systems via Wirtinger-based double integral inequality," Automatica. A Journal of IFAC, the International Federation of Automatic Control, vol. 55, pp. 204-208, 2015.
[9] Y. Liu and M. Li, "Improved robust stabilization method for linear systems with interval time-varying input delays by using Wirtinger inequality," ISA Transactions, vol. 56, pp. 111-122, 2015.

[10] J.-J. Hui, X.-Y. Kong, H.-X. Zhang, and X. Zhou, "Delaypartitioning approach for systems with interval time-varying delay and nonlinear perturbations," Journal of Computational and Applied Mathematics, vol. 281, pp. 74-81, 2015.

[11] J. An, Z. Li, and X. Wang, "A novel approach to delay-fractionaldependent stability criterion for linear systems with interval delay," ISA Transactions, vol. 53, no. 2, pp. 210-219, 2014.

[12] W. Zhang, X.-S. Cai, and Z.-Z. Han, "Robust stability criteria for systems with interval time-varying delay and nonlinear perturbations," Journal of Computational and Applied Mathematics, vol. 234, no. 1, pp. 174-180, 2010.

[13] K. Ramakrishnan and G. Ray, "Delay-range-dependent stability criterion for interval time-delay systems with nonlinear perturbations," International Journal of Automation and Computing, vol. 8, no. 1, pp. 141-146, 2011.

[14] J. J. Hui, H. X. Zhang, F. Meng, and X. Zhou, "Improved delay-rangedependent stability criteria for systems with interval timevarying delay and nonlinear perturba- tions," International Journal of Computer Science Issues, vol. 2, pp. 131-137, 2013.

[15] X. Zhou, H. Zhang, X. Hu, J. Hui, and T. Li, "Improved results on robust stability for systems with interval time-varying delays and nonlinear perturbations," Mathematical Problems in Engineering, vol. 2014, Article ID 898260, 7 pages, 2014.

[16] Y.-Y. Cao and J. Lam, "Computation of robust stability bounds for time-delay systems with nonlinear time-varying perturbations," International Journal of Systems Science, vol. 31, no. 3, pp. 359-365, 2000.

[17] Q.-L. Han, "Robust stability for a class of linear systems with time-varying delay and nonlinear perturbations," Computers \& Mathematics with Applications, vol. 47, no. 8-9, pp. 1201-1209, 2004.

[18] Z. Zuo and Y. Wang, "New stability criterion for a class of linear systems with time-varying delay and nonlinear perturbations," IEE Proceedings. Control Theory \& Applications, vol. 153, no. 5, pp. 623-626, 2006.

[19] Y. Chen, A. Xue, R. Lu, and S. Zhou, "On robustly exponential stability of uncertain neutral systems with time-varying delays and nonlinear perturbations," Nonlinear Analysis. Theory, Methods \& Applications, vol. 68, no. 8, pp. 2464-2470, 2008.

[20] F. Qiu, B. Cui, and Y. Ji, "Further results on robust stability of neutral system with mixed time-varying delays and nonlinear perturbations," Nonlinear Analysis: Real World Applications, vol. 11, no. 2, pp. 895-906, 2010.

[21] O. M. Kwon, J. H. Park, and S. M. Lee, "On robust stability criterion for dynamic systems with time-varying delays and nonlinear perturbations," Applied Mathematics and Computation, vol. 203, no. 2, pp. 937-942, 2008.

[22] R. Rakkiyappan, P. Balasubramaniam, and R. Krishnasamy, "Delay dependent stability analysis of neutral systems with mixed time-varying delays and nonlinear perturbations," Journal of Computational and Applied Mathematics, vol. 235, no. 8, pp. 2147-2156, 2011.

[23] J. Sun, G. P. Liu, J. Chen, and D. Rees, "Improved delay-rangedependent stability criteria for linear systems with time-varying delays," Automatica, vol. 46, no. 2, pp. 466-470, 2010.

[24] Y. Liu, L.-S. Hu, and P. Shi, "A novel approach on stabilization for linear systems with time-varying input delay," Applied 
Mathematics and Computation, vol. 218, no. 10, pp. 5937-5947, 2012.

[25] H. Shao, "New delay-dependent stability criteria for systems with interval delay," Automatica, vol. 45, no. 3, pp. 744-749, 2009.

[26] X.-L. Zhu, Y. Wang, and G.-H. Yang, "New stability criteria for continuous-time systems with interval time-varying delay," IET Control Theory \& Applications, vol. 4, no. 6, pp. 1101-1107, 2010.

[27] M. Tang, Y.-W. Wang, and C. Wen, "Improved delay-rangedependent stability criteria for linear systems with interval time-varying delays," IET Control Theory \& Applications, vol. 6, no. 6, pp. 868-873, 2012.

[28] P. Park, J. W. Ko, and C. Jeong, "Reciprocally convex approach to stability of systems with time-varying delays," Automatica, vol. 47, no. 1, pp. 235-238, 2011.

[29] W. Qian and J. Liu, "New stability analysis for systems with interval time-varying delay," Journal of the Franklin Institute, vol. 350, no. 4, pp. 890-897, 2013.

[30] W. Qian, S. Cong, T. Li, and S. Fei, "Improved stability conditions for systems with interval time-varying delay," International Journal of Control, Automation and Systems, vol. 10, no. 6, pp. 1146-1152, 2012. 


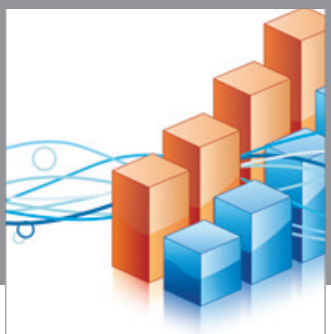

Advances in

Operations Research

vatem alat4

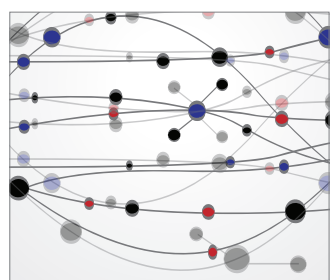

\section{The Scientific} World Journal
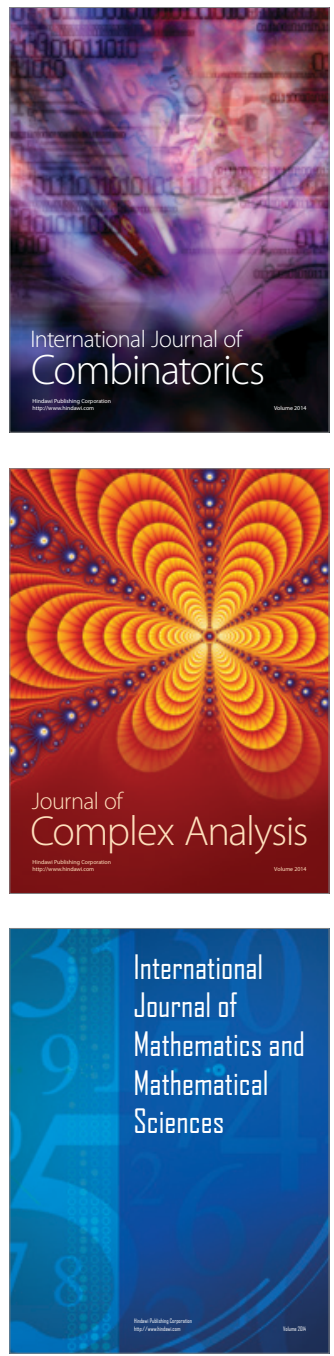
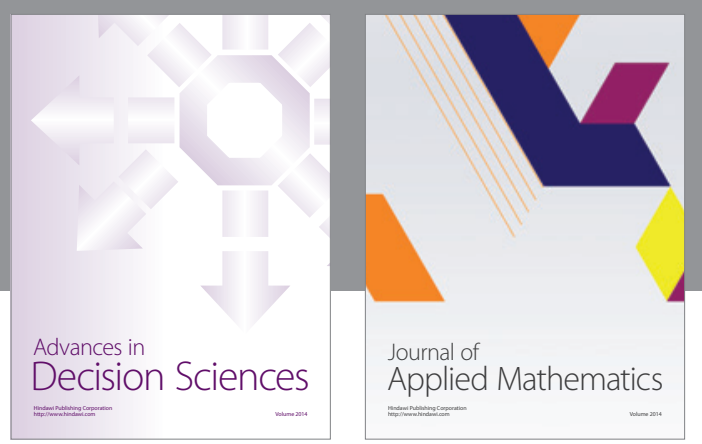

Algebra

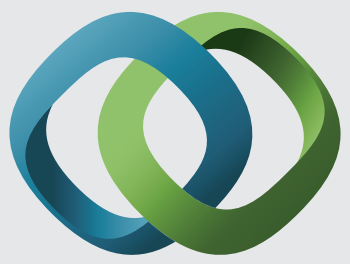

\section{Hindawi}

Submit your manuscripts at

https://www.hindawi.com
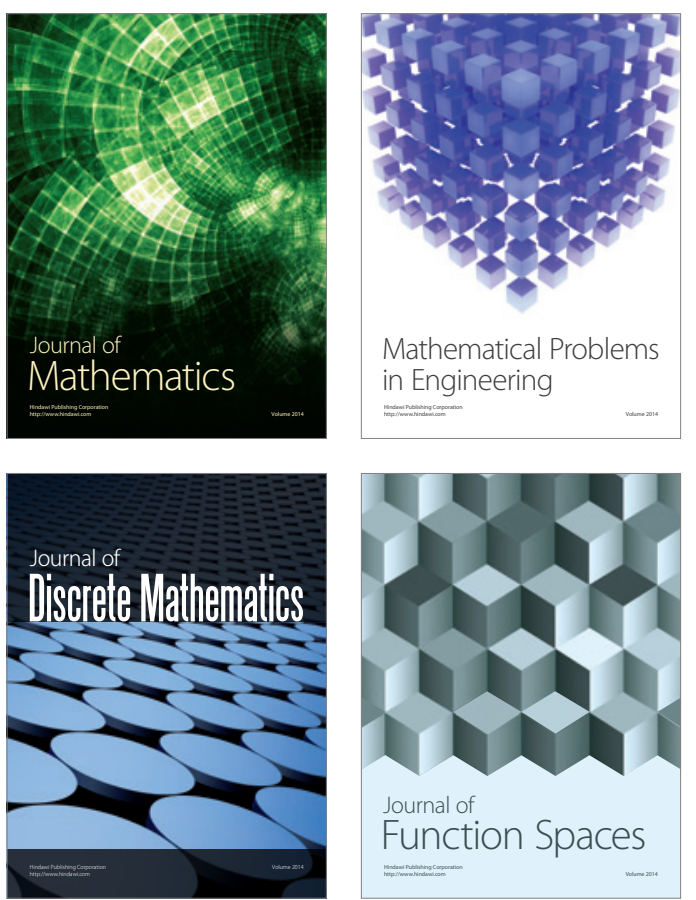

Mathematical Problems in Engineering
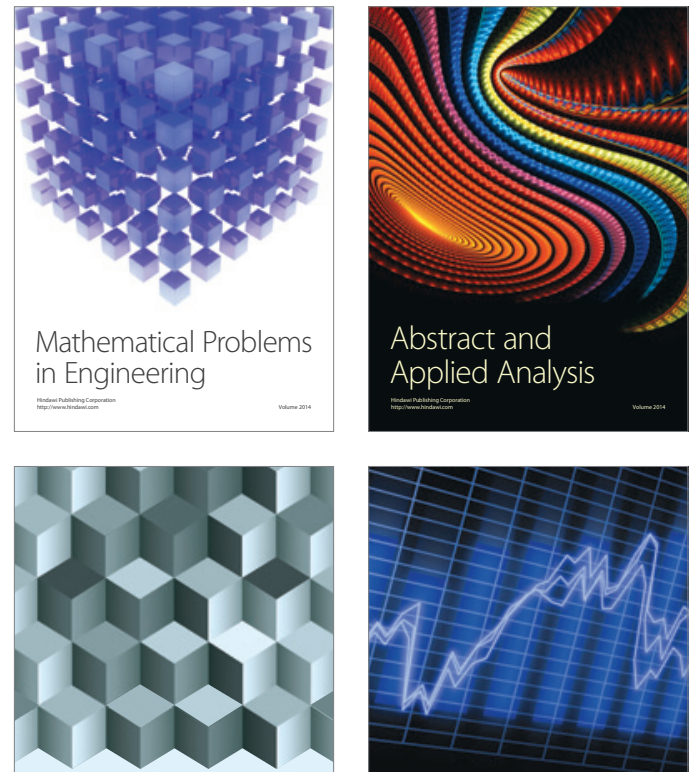

Journal of

Function Spaces

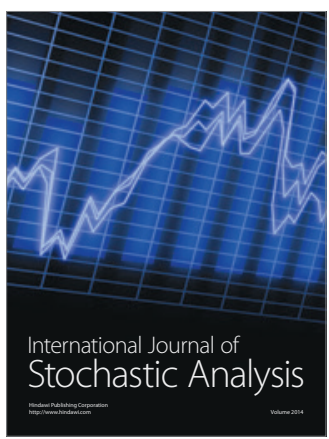

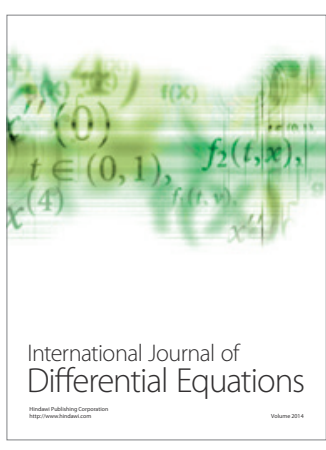
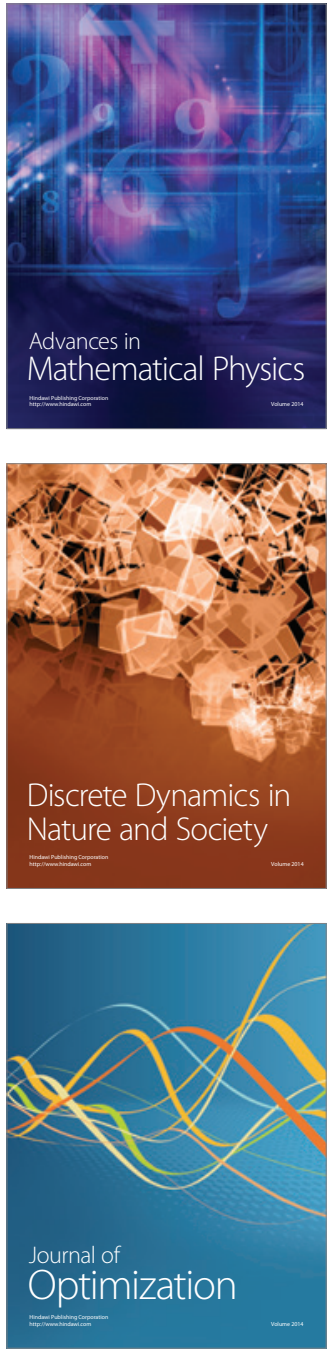